\title{
Graphene as intermediate phase in fullerene and carbon nanotube growth: A Young-Laplace surface-tension model
}

\author{
Vinay Gupta ${ }^{\text {a) }}$ \\ Physics of Energy Harvesting Division, National Physical Laboratory, K. S. Krishnan Marg, \\ New Delhi 110012, India
}

(Received 4 August 2010; accepted 8 October 2010; published online 3 November 2010)

\begin{abstract}
Growth mechanism of carbon nanotubes using arc-discharge are not fully understood up to now. Here, I show that the formation of carbon nanotubes and fullerene in a pure carbon arc in helium atmosphere may involve graphene bubbles. Electron microscopy shows the graphene bubbles formation at the anode surface. The growth of fullerene and nanotubes can occur from these bubbles due to a large pressure difference $\left(10^{3}-10^{4}\right.$ bar) at the anode interface and can be understood in terms of Young-Laplace law of surface tension. This model can explain the helium pressure dependence of fullerene and carbon nanotube formation. (C) 2010 American Institute of Physics. [doi:10.1063/1.3509403]
\end{abstract}

Despite two decades of intense studies, ${ }^{1-9}$ the growth mechanism of multiwalled carbon nanotubes (MWCNTs) (Ref. 2) in arc-discharge is not well understood. In the solid phase theory ${ }^{3}$ for the formation of MWCNT in arc-discharge, put forward in 1994, it was assumed that carbon in the vapor phase condenses onto the cathode in the form of fullerene sootlike material, acting as nanotube seeds. But this could not be established as to why the fullerene soot evolves into carbon nanotubes rather than nanoparticles when heated in arc? The gas phase theory, ${ }^{5}$ put forward in 1995, argued that nanotubes and nanoparticles form in the region of arc next to the cathode surface in isotropic manner. The key assumption of the gas phase theory was the formation of open ended structures, considered to be the seeds for nanotube growth. However, almost all MWCNTs are founded to be capped at the ends. The most recent liquid phase model, ${ }^{7}$ put forward in 2005, was a variant of solid state model with an idea that carbon nanotubes are formed in the interior of liquid carbon globules at the cathode. During the course of present investigation, it was observed that the liquid carbon globules are actually the left over impurity at arc-discharge plasma turn off point. Such globules were not observed when the electrodes were taken apart from each other without turning off the arc-discharge plasma (Fig. S1, Ref. 14). Therefore, there is currently no satisfactory understanding of the formation process of carbon nanotubes. The problem might be that the entire modeling process of nanotubes starts off from, and is dominated by the preassumption that the nucleation and growth of arc-discharge MWCNTs occur on the cathode. Here, based upon electron microscopy observation, I present a model that the formation of not only carbon nanotubes but also fullerene occur from the bubbling of graphene film that are formed on the anode surface by the unzipped graphite under extreme temperature and pressure conditions.

In this work, fullerenes and MWCNTs were synthesized in the electric arc-discharge apparatus (see Ref. 14 for details). The SEM images of the cathode deposit [Fig. 1(a)] shows columns of MWCNTs (few millimeter long and $\sim 0.1 \mathrm{~mm}$ wide, arranged parallel to the cylindrical axis. The formation of the column structures starts right from the base

${ }^{\text {a)} E l e c t r o n i c ~ m a i l: ~ d r v i n a y g u p t a @ ~ @ e t s c a p e . n e t . ~}$ of the deposit as shown by an arrow in Fig. 1(a). This contravenes the previous models that MWCNTs should be randomly oriented, at least on the base part of the cathode. The cross section of a broken column [inset of Fig. 1(a)] depicts that individual MWCNTs are highly oriented along to the cylindrical axis throughout the column structure. The extremely organized nature of the MWCNTs within the columns indicates that the formation process of carbon nanotubes is not chaotic as thought earlier. Figures 1(b)-1(d) shows the SEM and transmission electron microscopy (TEM) images of the anode surface after arc-discharge. It was observed that there exists a bright surface with silver luster that can be easily seen by scratching a part of the surface [shown by an arrow, left inset of Fig. 1(b)]. The bright part is composed of spherical bubbles [Fig. 1(b) and the right inset of Fig. 1(b)], which are formed up to several tenth micrometers depth into the anode and can be observed on the inside surface of hollow electrodes [Fig. 1(c)]. TEM image shows that bubbles are composed of multilayer graphenes [Fig. 1(d)]. On the other hand, the black part of the anode [left inset of Fig. 1(c)] contained MWCNTs as shown in the inset of Fig. 1(d). High resolution TEM of the tip area of a MWCNT is shown in Fig. 1(e). The tip of the MWCNT indicates that the growth of this nanotube started with the formation of fullereneslike structures of 1 and $2 \mathrm{~nm}$. A $5 \mathrm{~nm}$ seed fullerene-tube structure, encapsulated into a MWCNT is also shown in Fig. 1(f). Note that the $1 \mathrm{~nm}$ fullerene is nearly semispherical, whereas the 2 and $5 \mathrm{~nm}$ structures are in tube shape.

Graphene bubbles ${ }^{10}$ might play a key role in the nucleation and growth of fullerene and carbon nanotubes. This model describes the formation of fullerene and carbon nanotubes based on the simple concept of Young-Laplace surface tension, assuming that they are frozen graphene bubbles that are formed under specific temperature and pressure conditions given in the graphite phase-diagram [Fig. 2(a), taken from Ref. 10]. According to Dower et al., ${ }^{11}$ as arc-discharge is initiated, anode surface temperature reaches $4500-5000 \mathrm{~K}$ in nanoseconds (heating rate $\sim 10^{11} \mathrm{~K} / \mathrm{s}$ ) and due to that, the anode surface internal pressure jumps to $\sim 10^{4}$ bar [dotted curve in Fig. 2(a)] whereas the external applied helium pressure is $<1$ bar. Consequently, weak graphite Van der Waals 


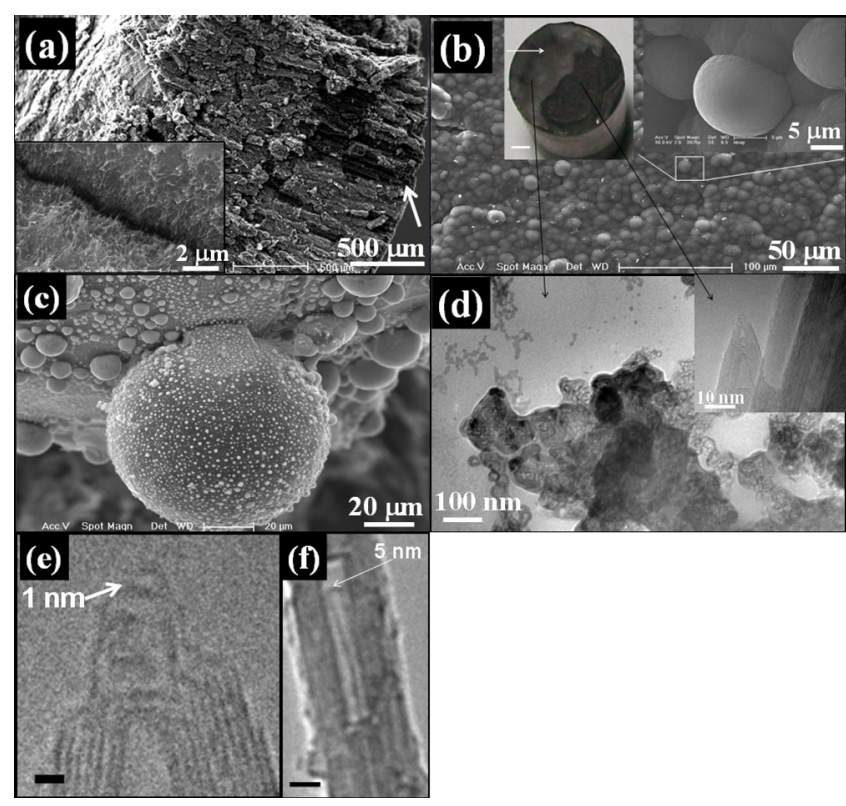

FIG. 1. (Color online) Electron microscopy images of cathode and anode after arc-discharge. (a) SEM of cross sectional view of cathode along the cylindrical axis. (Inset) SEM of interior of a column of cathode. (b) SEM of surface of the anode. The left inset show the optical image of anode surface. The right inset shows the close up image of graphene bubbles. (c) SEM image of the interior surface of a hollow anode after arc discharge. (d) TEM of the surface of anode. (e) TEM image of the cap of a MWCNT (scale bar $1 \mathrm{~nm}$ ) and (f) of a MWCNT showing a $5 \mathrm{~nm}$ graphene bubble.

bonding is unzipped to graphene long before it can melt to amorphous carbon. At the melting point of graphite, the $\mathrm{C}-\mathrm{C}$ bonding become extremely weak akin to hydrogen bonding and the characteristics of graphene film become similar to water film. ${ }^{12}$

According to the Young-Laplace law of surface tension, the formation of graphene bubbles from graphene film can be due to the pressure difference $\Delta P$ created at the interface of graphene, described by the equation; ${ }^{13}$

$$
\Delta P=\gamma_{L}\left(\frac{1}{r_{1}}+\frac{1}{r_{2}}\right) .
$$

Here, $r_{1}$ and $r_{2}$ are radii of curvature and $\gamma_{L}$ is surface tension. For a droplet (semibubble), $r_{1}=r_{2}=r_{b}$, the Eq. (1) leads to,

$$
\Delta P=\frac{2 \gamma_{L}}{r_{b}} .
$$

The parameters $\Delta P\left(P_{\mathrm{i}}-P_{\mathrm{o}}\right), \gamma_{L}$, and $r_{b}$, given in Eq. (2) are illustrated in Fig. 2(b). The implication of the YoungLaplace equation is that the radius of a bubble and $\Delta P$ are interdependent. In the case of large size bubble of water, the required $\Delta P$ is subtle but an enormous $\Delta P$ is required as the bubble size approaches the molecular size [Fig. 2(c)]. It is remarkable that the pressure difference requirement to form a water bubble of the size of fullerene is $\sim 10^{4}$ bar as calculated from Fig. 2(c). That is exactly the pressure difference at the anode surface when fullerenes are generated in arcdischarge at $\sim 5000 \mathrm{~K}$, according to the experimentally observed $\mathrm{P}-\mathrm{T}$ curve of a graphite anode in the phase-diagram [Fig. 2(a)]. Since fullerene bubbles are formed in nearly vacuum conditions, offering little external pressure, the pressure difference at the anode surface is same as the internal pressure, i.e., $\sim 10^{4}$ bar. For $\Delta P=10^{4}$ bar and $r_{b}=0.35 \mathrm{~nm}$

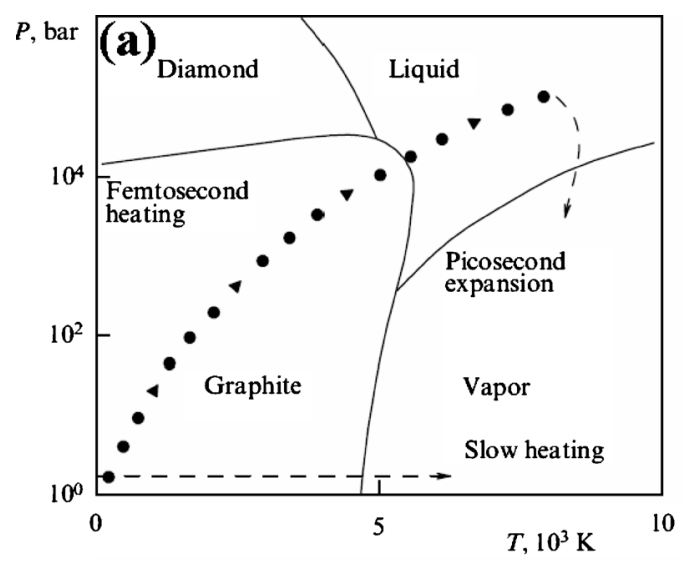

(b)
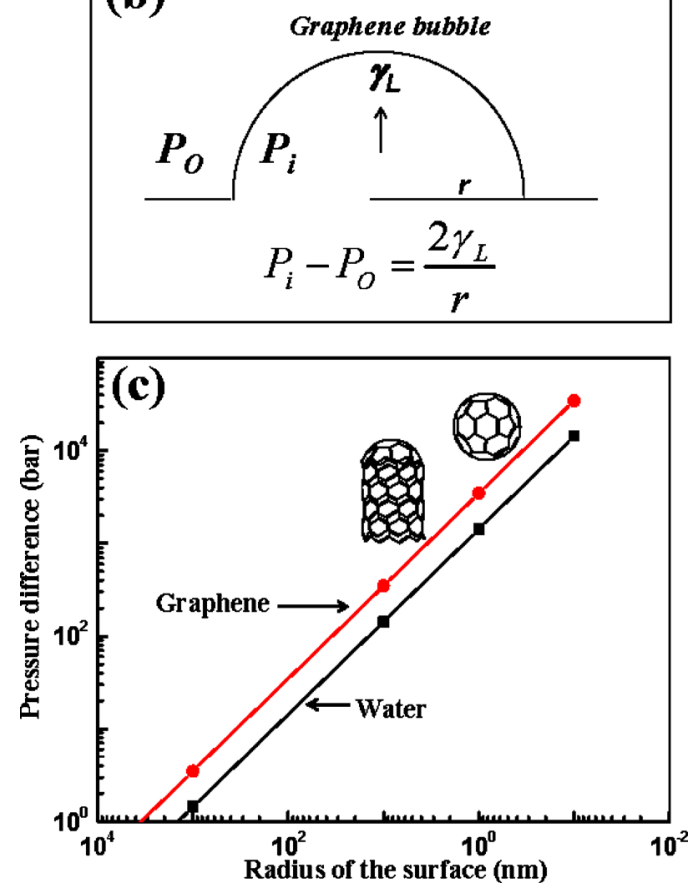

FIG. 2. (Color online) Young-Laplace surface tension and the phase diagram of graphite. (a) Equilibrium phase diagram of carbon (from Ref. 11). (b) Illustration of the bubble formation from a graphene and the parameters involved $\left(P_{\mathrm{o}}\right.$ is outside pressure, $P_{\mathrm{i}}$ is inside pressure) (c) dependence of radius of the of water and graphene droplets on the pressure difference at the interface.

(fullerene radius), $\gamma_{L}$ is roughly $0.175 \mathrm{~N} \mathrm{~m}^{-1}$, which is the surface tension of graphene film. Note that the surface tension value of graphene is close to that of water $\left(\gamma_{L}\right.$ $=0.072 \mathrm{~N} \mathrm{~m}^{-1}$ ). From the surface tension value of graphene film, the relationship between the radius of graphene bubble and $\Delta P$ is given in Fig. 2(c).

We have seen in Figs. 1(e) and 1(f) that as the size of the fullerene increases, its shape changes to tube like structure. The calculations from Fig. 2(c) show that the value of $\Delta P$ for the formation of a $1 \mathrm{~nm}$ radius fullerene is $3.5 \mathrm{kbar}$. This $\Delta P$ value requires an external $P_{\mathrm{He}}$ of several thousand bars at the surface, which is not possible considering that the applied external $P_{\mathrm{He}}$ is only around 700 mbar during carbon nanotube growth. Even if one considers that helium pressure is enhanced at the surface of the anode at $5000 \mathrm{~K}$ (pressure $\alpha$ temperature), an increase of $6.5 \mathrm{kbar}$ is still too high. So the formation of fullerenes of that size in arc-discharge is not observed because it violates the Young-Laplace Eq. (1). Therefore, the $\Delta P$ at the anode remains much higher than 

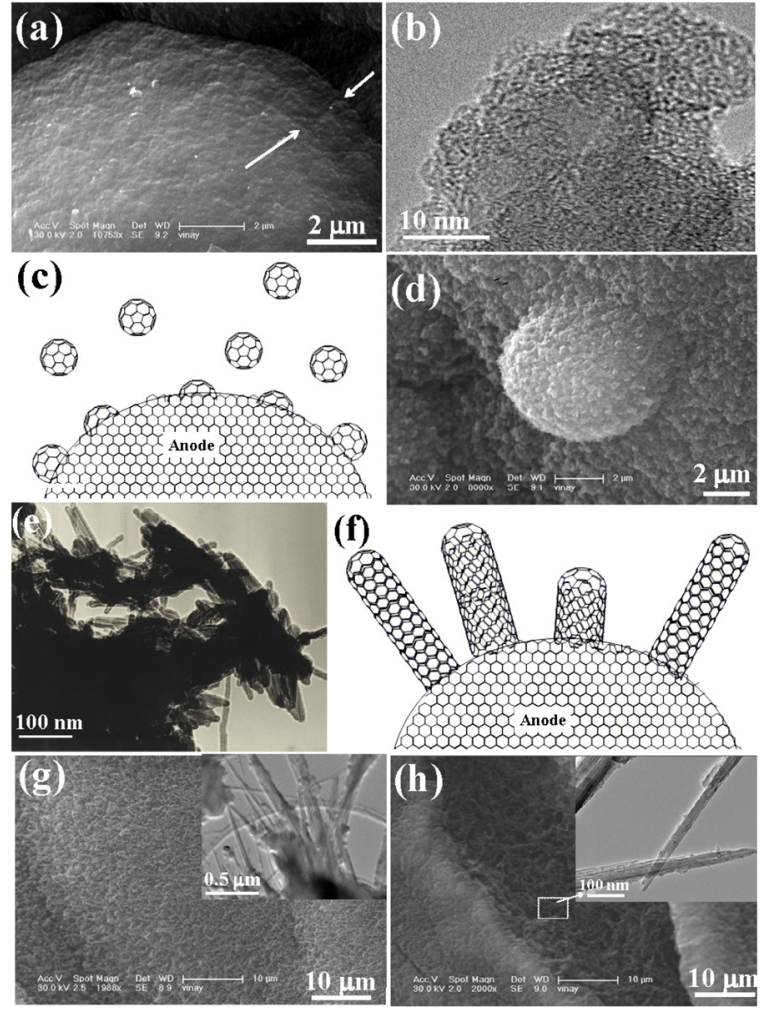

FIG. 3. (Color online) Electron microscopy imaging of evolution process of [(a)-(c)] fullerene and [(d)-(h)], carbon nanotube after arc-discharge. (a) SEM of anode surface. (b) TEM of the anode surface at $P_{\mathrm{He}} \sim 10$ mbar. (c) illustration of fullerene formation process. [(d), (g), and (h)] SEM images of anode surface. [(e)] TEM images of the anode surface at $P_{\mathrm{He}} \sim 700 \mathrm{mbar}$; different steps of MWCNT formation are shown. (e) Illustration of carbon nanotubes formation process.

required for the formation of a large diameter $(>1 \mathrm{~nm})$ fullerenes, so fullerenes structure grow unidirectional instead of radial direction in order to conserve Young-Laplace equation as well as balancing the pressure and that results in the formation of elongated fullerenes leading to nanotubes. The length of a nanotube depends upon the equilibrium condition of a bubble. The natural hexagonal arrangement of atoms on graphene surface provides strength against any destabilization. It is evident that the timeframe for the formation of MWCNTs is at least an order of magnitude higher than in the case of fullerene. It was experimentally observed that the rate of consumption of anode at $P_{\mathrm{He}} \sim 700 \mathrm{mbar}$ is eight to ten times slower than at $P_{\mathrm{He}} \sim 20$ mbar. The illustration of fullerenes and nanotubes in comparison with the formation of soap bubbles from a soap film is shown in Fig. S2, (Ref. 14). Moreover, TEM observations of the graphene obtained from the surface indicates the presence of single layers at low pressure and multilayers at high helium pressure. Since the internal pressure is much larger than the external pressure, large changes in the tube diameter is not likely but a change in the $1-10$ is possible.

The SEM image of anode surface after arc discharge at $P_{\mathrm{He}} \sim 10 \mathrm{mbar}[$ Fig. 3(a)] indicates the presence of graphene layers that are distinguishable from each other (shown by arrows). TEM image shows the presence of $\mathrm{C}_{60}$-like structure on the surface [Fig. 3(b)], which is in good agreement with the $r_{b}-\Delta P$ curve of the graphene [Fig. 2(c)]. The process of the formation of fullerenes is illustrated in Fig. 3(c). As the $P_{\mathrm{He}}$ is increased to $\sim 700 \mathrm{mbar}$, surface morphology of the anode is completely changed and evolution of carbon nanotubes is observed. The step-wise sequence of the formation of MWCNT is illustrated in Figs. 3(d)-3(h). Onedimensional structure can be clearly seen to be protruding from bubblelike surface of anode in Fig. 3(d). TEM image indicate that the protruding structure is composed of MWCNTs (length $=100-200 \mathrm{~nm}$ ) [Fig. 3(f) and Fig. S3, Ref. 14]. The process of crystallization of graphene into MWCNTs is illustrated in Fig. 3(f). A further growth [step-II, Fig. 3(g)] results in the filling of entire surface with MWCNTs. After that, the bubblelike surface of the anode breaks down [step-III, Fig. 3(h)] and the formed MWCNTs are then transferred to cathode. This process is repeated continuously during the arc-discharge. During the transfer process, a majority of MWCNTs, particularly at the outer region, do not survive and are converted to graphitic structure. Therefore the cathode deposit contains a thick graphitic shell. TEM image of the MWCNTs is shown in the inset of Fig. 3(h). The formation of branch structure of MWCNTs is explained in Fig. S4, (Ref. 14).

In summary, I have shown that once the graphite is converted to graphene at the anode surface, the pressure difference across the graphene film surface results in the formation of bubbles. Since the size and shape of the bubble depends upon the pressure difference, it results in the formation of fullerenes and carbon nanotubes. It may possible to make different types of graphite composites as anode which may results into several different atoms encapsulated into fullerenes and carbon nanotubes during the synthesis process. It can be predicted that the use of graphene electrode as anode should give much better yield of fullerenes and MWCNTs.

${ }^{1}$ S. Iijima, Nature (London) 354, 56 (1991)

${ }^{2}$ T. W. Ebbesen, and P. M. Ajayan, Nature (London) 358, 220 (1992).

${ }^{3}$ P. J. F. Harris, S. C. Tsang, J. B. Claridge, and M. L. H. Green, J. Chem. Soc., Faraday Trans. 90, 2799 (1994).

${ }^{4}$ D. T. Colbert, J. Zhang, S. M. McClure, P. Nikolaev, Z. Chen, J. H. Hafner, D. W. Owens, P. G. Kotula, C. B. Carter, J. H. Weaver, A. G. Rinzler, and R. E. Smalley, Science 266, 1218 (1994).

${ }^{5}$ E. G. Gamaly and T. W. Ebbesen, Phys. Rev. B 52, 2083 (1995).

${ }^{6}$ D. Zhou and L. Chow, J. Appl. Phys. 93, 9972 (2003).

${ }^{7}$ W. A. de Heer, P. Poncharal, C. Berger, J. Gezo, Z. Song, J. Bettini, and D. Ugarte, Science 307, 907 (2005).

${ }^{8}$ P. J. F. Harris, Carbon 45, 229 (2007).

${ }^{9}$ P. J. F. Harris, Carbon Nanotube Science: Synthesis, Properties and applications (Cambridge University Press, Cambridge, UK, 2009).

${ }^{10}$ N. Levy, S. A. Burke, K. L. Meaker, M. Panlasigui, A. Zettl, F. Guinea, A. H. Castro Neto, and M. F. Crommie, Science 329, 544 (2010).

${ }^{11}$ M. C. Downer, H. Ahn, D. H. Reitze, and X. Y. Wang, Int. J. Thermophys 14, 361 (1993).

${ }^{12}$ The observed value to resistivity of graphite at the solid-liquid transition is $\sim 600 \mu \Omega \mathrm{cm}$, which is a sign of graphene state and not an amorphous carbon state (Ref. 11).

${ }^{13}$ H. Jügen and M. Kappl, Surface and Interfacial Forces (Wiley-VCH, Weinheim, Germany, 2010).

${ }^{14}$ See supplementary material at http://dx.doi.org/10.1063/1.3509403 for scanning electron microscopy (SEM) images (Fig. S1), optical images (Fig. S2), TEM image (Fig. S3), and schematic diagram (Fig. S4). 